\title{
Effect of Integrated Nutrient Management (INM) on Physico-Chemical Properties of Soil, Available Content and Nutrient Uptake by Okra (Abelmoschus esculentus)
}

\author{
Veer Bhan Singh and Anil Kumar Tiwari*
}

Department of Horticulture, Faculty of Agriculture \& Allied Industries,
Rama University Kanpur (U.P.) India

*Corresponding author

\begin{abstract}
A B S T R A C T
The present investigation was conducted at Research Farm, Rama University, Kanpur in 2018. Total 11 treatments, each replicated thrice in RBD. Soil fertility status after the crop

Keywords

Okra. INM,

Nutrient uptake,

Physio-chemical, Soil

Article Info

Accepted:

04 February 2019

Available Online:

10 March 2019 harvested revealed that the available nitrogen is maximum in $\mathrm{T}_{4}\left(315.72 \mathrm{~kg} \mathrm{ha}^{-1}\right)$ and minimum in $\mathrm{T}_{11}\left(258.84 \mathrm{~kg} \mathrm{ha}^{-1}\right)$. Available phosphorus is maximum $\left(22.16 \mathrm{~kg} \mathrm{ha}^{-1}\right)$ in $\mathrm{T}_{4}$ and minimum $\left(15.81 \mathrm{~kg} \mathrm{ha}^{-1}\right)$ in $\mathrm{T}_{11}$. Whereas available potassium is maximum $(210.36 \mathrm{~kg}$ $\left.\mathrm{ha}^{-1}\right)$ in $\mathrm{T}_{4}$ and minimum $\left(179.35 \mathrm{~kg} \mathrm{ha}^{-1}\right)$ in $\mathrm{T}_{11}$ treatment. The use of inorganic fertilizers and organic manures or micronutrients was found significant ranged between 1.51 to 1.63 percent. The higher $\mathrm{P}_{2} \mathrm{O}_{5}$ content $(0.63 \%)$ was observed in treatment $\mathrm{T}_{4}$ which was at par with the treatments $\mathrm{T}_{2}(0.62), \mathrm{T}_{3}(0.61)$ and $\mathrm{T}_{1}(0.60)$ where as the lower phosphorus content $(0.50 \%)$ was observed in treatment $\mathrm{T}_{11}$. The maximum $\mathrm{K}_{2} \mathrm{O}$ content was found in treatment $\mathrm{T}_{4}(1.46 \%)$, during the crop study period. The minimum $\mathrm{K}_{2} \mathrm{O}$ content was found in treatment $\mathrm{T}_{11}(1.30 \%)$. The nitrogen uptake was significantly highest $(2.22 \mathrm{t} / \mathrm{ha})$ in the treatment $\mathrm{T}_{4}$ over all other treatments. The least uptake of nitrogen was found in the treatment $\mathrm{T}_{11}(0.51 \mathrm{t} / \mathrm{ha})$. Phosphorus $\left(\mathrm{P}_{2} \mathrm{O}_{5}\right)$ uptake in course of investigation, the higher $\mathrm{P}_{2} \mathrm{O}_{5}$ uptake in pod was $0.85 \mathrm{~kg} / \mathrm{ha}\left(\mathrm{T}_{4}\right)$ and minimum uptake in pod was $0.17 \mathrm{~kg} / \mathrm{ha}$ in $\mathrm{T}_{11}$. $\mathrm{K}$ uptake in pods was $1.99 \mathrm{~kg} / \mathrm{ha}\left(\mathrm{T}_{4}\right)$ and lowest uptake in pods was $0.43 \mathrm{~kg} / \mathrm{ha}$ in $\mathrm{T}_{11}$.
\end{abstract}

\section{Introduction}

Okra is one of the most important vegetable crop grown extensively throughout the country during summer and rainy season due to its high adaptability over a wide range of environmental conditions. It is widely adapted vegetable in Indian kitchens and can be grown though out the year. As a vegetable in tender stage, okra is nutritious and it finds an important place in the Indian dietary. Besides the utility of its tender green fingers as a vegetable, it is used in soups and curries. Green pods are rich source of Iodine, vitamin A, B and C. The stems and roots of okra can also be used in paper industry. India ranks first in the world with the production of 3.5 million tonnes $(70 \%$ of the total world production) from an area of 0.35 million ha. 
Organic manures not only balance the nutrient supply but also improve the physical and chemical quality of Okra, being a short duration vegetable crop, its growth, yield and quality are largely influenced by the application of fertilizers. It requires proper and sufficient $\mathrm{N}, \mathrm{P} \& \mathrm{~K}$ for regular fruiting and subsequent pickings (Premsekhar and Rajashree, 2009). It is well known fact that organic manures, inorganic fertilizers and micronutrients are essential to increase the yield of vegetable crops. Owing to increased cost of fertilizers, their short supply and sustainability issues, it is felt essential to reduce the dependence on chemical fertilizers. Therefore, it is imperative that chemical fertilizers, organic manures as well as micronutrients are utilized properly and effectively not only as source of the nutrients but also for increasing nutrients use efficiency without adversely disturbing the soil health.

\section{Materials and Methods}

The present investigation was conducted in Research Farm, Faculty of Agriculture and Allied Industries, Rama University, Kanpur (U.P.) during Rabi season of 2017-18. The experimental farm falls under the Indogangetic alluvial tract of Central Uttar Pradesh. The farm was well laid out and irrigated by tube well.

The experiments were conducted in a Randomized Block Design replicated thrice. There were eleven treatments involving different organic manures along with chemical fertilizers. The seeds were sown at the spacing of $45 \mathrm{~cm}$ between rows and $30 \mathrm{~cm}$ within the row. The field was irrigated immediately after sowing. Bold and healthy seeds of Okra cv. Arka Anamika were dibbled at two seeds per hill. Gap filling was done after one week. Before sowing, Vermicompost, Farm Yard Manure and Poultry manure was applied to the soil as a basal dose as per the treatments. Nitrogen was applied in the form of urea in two equal splits i.e., as basal dose and subsequent dose at flowering stage. Phosphorus and potassium were applied as a basal dose only in the form of SSP and MOP respectively. The soil of the experimental field was well leveled. The fertility status and textural class of the soil were judged by chemical, physical and mechanical analysis. For purpose, soil samples were taken randomly from 5 places of experimental plot from the depth of $15 \mathrm{~cm}$. just before sowing and fertilizer application. The soil of these samples was mixed thoroughly and a representative soil sample was drawn. The quantity of soil sample was reduced to about one $\mathrm{kg}$ through quartering technique.

\section{Results and Discussion}

\section{Soil of experimental field}

This sample was subjected to mechanical and chemical analysis. The method employed in both the analysis and results obtained are presented in Table 1.

\section{Available N, P, K (Kg/ha)}

The initial status of available $\mathrm{N}$ in soil was $230 \mathrm{~kg} / \mathrm{ha}$. The data subjected to statistically analysis showed that it was significantly affected by the different INM with organic treatments during experimental periods. During crop period, among the treatments, the maximum value of $315.72 \mathrm{~kg} / \mathrm{ha}$ available $\mathrm{N}$ was recorded in treatment $\mathrm{T}_{4}(50 \% \mathrm{RDF}$ inorganic $+50 \% \mathrm{~N}$ through Vermicompost (VC)), $\mathrm{T}_{2}(50 \% \mathrm{RDF}$ inorganic $+50 \% \mathrm{~N}$ through Farm yard manure (FYM)) and $\mathrm{T}_{3}$ (50\% RDF inorganic $+50 \% \mathrm{~N}$ through Poultry manure (PM)) which was at par with each other. The minimum available $\mathrm{N}$ was recorded in treatment $\mathrm{T}_{11}$ consisting of control after harvest of okra crop. 
The initial status of available $\mathrm{P}$ was $40 \mathrm{~kg} / \mathrm{ha}$. The available $\mathrm{P}_{2} \mathrm{O}_{5}$ in soil after harvesting of okra crop was influenced significantly and it was interesting to note that available $\mathrm{P}_{2} \mathrm{O}_{5}$ level has increased. The data revealed that the values of available $\mathrm{P}_{2} \mathrm{O}_{5}$ varied from 15.81 to $22.16 \mathrm{~kg} / \mathrm{ha}$. The higher available $\mathrm{P}_{2} \mathrm{O}_{5}(22.16$ $\mathrm{kg} / \mathrm{ha}$ ) was noticed under the treatment $\mathrm{T}_{4}$ $(50 \% \mathrm{RDF}$ inorganic $+50 \% \mathrm{~N}$ through Vermicompost (VC)); while minimum (15.81 $\mathrm{kg} / \mathrm{ha}$ ) was recorded in treatment $\mathrm{T}_{11}$ (control).
The initial status of available $\mathrm{K}$ was 259 $\mathrm{kg} / \mathrm{ha}$. In case of available $\mathrm{K}_{2} \mathrm{O}$ in soil, after harvesting of okra crop, it was influenced significantly due to different INM. During the experimental period, the values pertaining to available $\mathrm{K}_{2} \mathrm{O}$ varied from 179.35 to 210.36 $\mathrm{kg} / \mathrm{ha}$. The maximum value $(210.36 \mathrm{~kg} / \mathrm{ha})$ was recorded under the treatment of $(50 \%$ $\mathrm{RDF}$ inorganic $+50 \% \mathrm{~N}$ through Vermicompost (VC) $\left(\mathrm{T}_{4}\right)$ and was at par with the treatments $T_{2}, T_{3}$ and $T_{1}$. The minimum content value $(179.35 \mathrm{~kg} / \mathrm{ha})$ was noticed under the treatment control $\left(\mathrm{T}_{11}\right)$ (Table 2-4).

Table.1 Physico-chemical properties of experimental field

\begin{tabular}{|c|c|c|c|c|}
\hline S.No. & Soil Properties & Values & Method of determination & Reference \\
\hline (A) & \multicolumn{4}{|c|}{ Mechanical Analysis } \\
\hline 1. & Coarse sand $(\%)$ & 0.75 & $\begin{array}{l}\text { International } \quad \text { Pipette } \\
\text { Method }\end{array}$ & Piper 1950 \\
\hline 2. & Fine sand $(\%)$ & 55.80 & $\begin{array}{l}\text { International Pipette } \\
\text { Method }\end{array}$ & Piper 1950 \\
\hline 3. & Silt (\%) & 23.50 & & Piper 1950 \\
\hline 4. & Clay $(\%)$ & 21.80 & $\begin{array}{l}\text { International } \\
\text { method }\end{array}$ & Piper 1950 \\
\hline 5. & Texture class & $\begin{array}{l}\text { Sandy } \\
\text { loam }\end{array}$ & USDA, Triangle & $\begin{array}{lll}\text { Soil } & \text { survey } \\
1975 & & \end{array}$ \\
\hline (B) & \multicolumn{4}{|l|}{ Physical Analysis } \\
\hline 1. & $\begin{array}{l}\text { Bulk density ( } \mathrm{mg} \mathrm{m}^{-} \\
3 \text { ) }\end{array}$ & 1.367 & $\begin{array}{l}\text { Core Cutter Sampler } \\
\text { Method }\end{array}$ & $\begin{array}{l}\text { USDA Handbook } \\
\text { (LA Richards 1954) }\end{array}$ \\
\hline (C) & \multicolumn{4}{|l|}{ Chemical Analysis } \\
\hline 1. & Organic Carbon $(\%)$ & 0.44 & $\begin{array}{l}\text { Walkley and Block } \\
\text { Rapid Titration Method }\end{array}$ & Jackson 1967 \\
\hline 2. & Available $\mathrm{N}\left(\mathrm{kg} \mathrm{ha}^{-1}\right)$ & 170.0 & $\begin{array}{l}\text { Alkaline Potassium Per } \\
\text { magnate Method }\end{array}$ & Jackson 1967 \\
\hline 3. & $\begin{array}{l}\text { Available } \\
\left.\mathrm{P}_{2} \mathrm{O}_{5}^{-1}\right)\end{array}$ & 17.8 & Olsen’s Method & Olsen et al., 1954 \\
\hline 4. & $\begin{array}{l}\text { Available } \quad \mathrm{K}_{2} \mathrm{O} \quad(\mathrm{kg} \\
\left.\mathrm{ha}^{-1}\right)\end{array}$ & 165.0 & $\begin{array}{l}\text { Flame } \quad \text { Photometer } \\
\text { Method }\end{array}$ & Olsen et al., 1954 \\
\hline 5. & $\mathrm{pH}$ & 7.5 & $\begin{array}{l}\text { Electronic } \\
\text { Electrode Method }\end{array}$ & Piper 1950 \\
\hline 6. & $\mathrm{EC}\left(\mathrm{dsm}^{-1}\right)$ & 0.25 & $\begin{array}{l}\text { Electrical Conductivity } \\
\text { Meter }\end{array}$ & Piper 1950 \\
\hline 7. & Zn (mg-ha- ${ }^{\mathbf{1}}$ ) & 1.1 & $\begin{array}{l}\text { Extracted by DTPA \& } \\
\text { Analysed on AAS }\end{array}$ & Piper 1950 \\
\hline
\end{tabular}


Table.2 Effect of integrated nutrient management on available $\mathrm{N}, \mathrm{P}$ and $\mathrm{K}\left(\mathrm{kg} \mathrm{ha}^{-1}\right)$ of Okra

\begin{tabular}{|c|c|c|c|c|}
\hline Treatment & Treatment Combination & $\begin{array}{l}\text { Available } \\
\text { N (kg/ha) }\end{array}$ & $\begin{array}{l}\text { Available } \\
\text { P (kg/ha) }\end{array}$ & $\begin{array}{l}\text { Available } \\
\text { k (kg/ha) }\end{array}$ \\
\hline $\mathbf{T}_{1}$ & $\begin{array}{l}100 \% \text { inorganic RDF (N:P:K- } \\
120: 60: 80)\end{array}$ & 287.92 & 20.91 & 199.07 \\
\hline $\mathbf{T}_{2}$ & $\begin{array}{l}50 \% \text { RDF inorganic }+50 \% \mathrm{~N} \\
\text { through Farm yard manure }(\mathrm{FYM})\end{array}$ & 290.29 & 21.49 & 204.48 \\
\hline $\mathbf{T}_{\mathbf{3}}$ & $\begin{array}{l}50 \% \text { RDF inorganic }+50 \% \mathrm{~N} \\
\text { through Poultry manure }(\mathrm{PM})\end{array}$ & 292.44 & 21.21 & 203.39 \\
\hline $\mathbf{T}_{4}$ & $\begin{array}{l}50 \% \text { RDF inorganic }+50 \% \mathrm{~N} \\
\text { through Vermicompost }(\mathrm{VC})\end{array}$ & 315.72 & 22.16 & 210.36 \\
\hline $\mathbf{T}_{5}$ & $\begin{array}{l}25 \% \text { RDF inorganic }+75 \% \mathrm{~N} \\
\text { through Farm yard manure }(\mathrm{FYM})\end{array}$ & 281.48 & 18.74 & 192.77 \\
\hline $\mathbf{T}_{6}$ & $\begin{array}{l}25 \% \text { RDF inorganic }+75 \% \mathrm{~N} \\
\text { through Poultry manure }(\mathrm{PM})\end{array}$ & 280.25 & 18.68 & 190.61 \\
\hline $\mathbf{T}_{7}$ & $\begin{array}{l}25 \% \text { RDF inorganic }+75 \% \mathrm{~N} \\
\text { through Vermicompost }(\mathrm{VC})\end{array}$ & 284.89 & 18.94 & 195.86 \\
\hline $\mathbf{T}_{8}$ & $\begin{array}{l}100 \% \mathrm{~N} \text { through Farm yard } \\
\text { manure }(\mathrm{FYM})(16.0 \mathrm{t} / \mathrm{ha})\end{array}$ & 270.63 & 17.65 & 186.24 \\
\hline $\mathbf{T}_{9}$ & $\begin{array}{l}100 \% \mathrm{~N} \text { through Poultry manure } \\
(\mathrm{PM})(5.0 \mathrm{t} / \mathrm{ha})\end{array}$ & 264.76 & 16.77 & 183.73 \\
\hline $\mathbf{T}_{10}$ & $\begin{array}{l}100 \% \mathrm{~N} \text { through Vermicompost } \\
(\mathrm{VC})(7.0 \mathrm{t} / \mathrm{ha})\end{array}$ & 275.68 & 17.99 & 188.15 \\
\hline $\mathbf{T}_{11}$ & $\begin{array}{l}\text { Control (without inorganic or } \\
\text { organic sources of nutrition) }\end{array}$ & 258.84 & 15.81 & 179.35 \\
\hline & C.D. & 7.09 & 3.85 & 5.75 \\
\hline & $\mathrm{SE}(\mathrm{m})$ & 2.39 & 1.30 & 1.94 \\
\hline & C.V. & 1.47 & 11.73 & 1.73 \\
\hline
\end{tabular}


Table.3 Effect of integrated nutrient management on $\mathrm{N}, \mathrm{P}$ and $\mathrm{K}\left(\mathrm{kg} \mathrm{ha}^{-1}\right)$ content of Okra

\begin{tabular}{|c|c|c|c|c|}
\hline Treatment & Treatment Combination & $\begin{array}{l}N \\
\text { content } \\
(\%)\end{array}$ & $\begin{array}{l}\text { P } \\
\text { content } \\
(\%)\end{array}$ & $\begin{array}{l}\text { K } \\
\text { content } \\
(\%)\end{array}$ \\
\hline $\mathbf{T}_{1}$ & $\begin{array}{ll}100 \% \quad \text { inorganic } & \text { RDF } \\
(\mathrm{N}: \mathrm{P}: \mathrm{K}-120: 60: 80) & \end{array}$ & 1.60 & 0.60 & 1.42 \\
\hline $\mathbf{T}_{2}$ & $\begin{array}{l}50 \% \text { RDF inorganic }+50 \% \\
\mathrm{~N} \text { through Farm yard } \\
\text { manure (FYM) }\end{array}$ & 1.62 & 0.62 & 1.45 \\
\hline $\mathbf{T}_{3}$ & $\begin{array}{l}50 \% \text { RDF inorganic }+50 \% \\
\mathrm{~N} \text { through Poultry manure } \\
(\mathrm{PM})\end{array}$ & 1.61 & 0.61 & 1.44 \\
\hline $\mathbf{T}_{4}$ & $\begin{array}{l}50 \% \text { RDF inorganic }+50 \% \\
\mathrm{~N} \text { through Vermicompost } \\
\text { (VC) }\end{array}$ & 1.63 & 0.63 & 1.46 \\
\hline $\mathbf{T}_{5}$ & $\begin{array}{l}25 \% \text { RDF inorganic }+75 \% \\
\mathrm{~N} \text { through Farm yard } \\
\text { manure (FYM) }\end{array}$ & 1.57 & 0.56 & 1.37 \\
\hline$T_{6}$ & $\begin{array}{l}25 \% \text { RDF inorganic }+75 \% \\
\mathrm{~N} \text { through Poultry manure } \\
(\mathrm{PM})\end{array}$ & 1.56 & 0.57 & 1.36 \\
\hline $\mathbf{T}_{7}$ & $\begin{array}{l}25 \% \text { RDF inorganic }+75 \% \\
N \text { through Vermicompost } \\
\text { (VC) }\end{array}$ & 1.58 & 0.58 & 1.40 \\
\hline $\mathbf{T}_{8}$ & $\begin{array}{ll}100 \% \mathrm{~N} \text { through } & \text { Farm } \\
\text { yard manure }(\mathrm{FYM}) & (16.0 \\
\text { t/ha) } & \end{array}$ & 1.54 & 0.54 & 1.33 \\
\hline$T_{9}$ & $\begin{array}{l}100 \% \mathrm{~N} \text { through Poultry } \\
\text { manure (PM) }(5.0 \mathrm{t} / \mathrm{ha})\end{array}$ & 1.53 & 0.53 & 1.31 \\
\hline$T_{10}$ & $\begin{array}{ll}100 \% \quad \mathrm{~N} & \text { through } \\
\text { Vermicompost } & (\mathrm{VC}) \\
\text { t/ha) } & (7.0\end{array}$ & 1.55 & 0.55 & 1.35 \\
\hline$T_{11}$ & $\begin{array}{l}\text { Control (without inorganic } \\
\text { or organic sources of } \\
\text { nutrition) }\end{array}$ & 1.51 & 0.50 & 1.30 \\
\hline & C.D. & 0.03 & 0.04 & 0.04 \\
\hline & SE(m) & 0.01 & 0.01 & 0.01 \\
\hline & C.V. & 0.97 & 4.02 & 1.65 \\
\hline
\end{tabular}


Table.4 Effect of integrated nutrient management on N, P and K uptake $\left(\mathrm{kg} \mathrm{ha}^{-1}\right)$ of Okra

\begin{tabular}{|c|c|c|c|c|}
\hline Treatment & Treatment Combination & $\begin{array}{l}\text { N } \\
\text { uptake } \\
\text { (kg/ha) }\end{array}$ & $\begin{array}{l}\text { P uptake } \\
\text { (kg/ha) }\end{array}$ & $\begin{array}{l}\text { K } \\
\text { uptake } \\
\text { (kg/ha) }\end{array}$ \\
\hline $\mathbf{T}_{1}$ & $\begin{array}{l}\text { 100\% inorganic RDF (N:P:K- } \\
120: 60: 80 \text { ) }\end{array}$ & 1.68 & 0.63 & 1.49 \\
\hline $\mathbf{T}_{2}$ & $\begin{array}{l}50 \% \text { RDF inorganic }+50 \% \mathrm{~N} \\
\text { through Farm yard manure } \\
(\text { FYM) }\end{array}$ & 2.00 & 0.76 & 1.79 \\
\hline $\mathbf{T}_{\mathbf{3}}$ & $\begin{array}{l}50 \% \text { RDF inorganic }+50 \% \mathrm{~N} \\
\text { through Poultry manure (PM) }\end{array}$ & 1.85 & 0.71 & 1.66 \\
\hline $\mathbf{T}_{4}$ & $\begin{array}{l}50 \% \text { RDF inorganic }+50 \% \mathrm{~N} \\
\text { through Vermicompost (VC) }\end{array}$ & 2.22 & 0.85 & 1.99 \\
\hline $\mathbf{T}_{5}$ & $\begin{array}{l}25 \% \text { RDF inorganic }+75 \% \mathrm{~N} \\
\text { through Farm yard manure } \\
(\text { FYM) }\end{array}$ & 1.15 & 0.42 & 1.01 \\
\hline$T_{6}$ & $\begin{array}{l}25 \% \text { RDF inorganic }+75 \% \mathrm{~N} \\
\text { through Poultry manure (PM) }\end{array}$ & 1.13 & 0.42 & 0.99 \\
\hline $\mathbf{T}_{7}$ & $\begin{array}{l}25 \% \text { RDF inorganic }+75 \% \mathrm{~N} \\
\text { through Vermicompost }(\mathrm{VC})\end{array}$ & 1.24 & 0.46 & 1.10 \\
\hline $\mathbf{T}_{8}$ & $\begin{array}{l}100 \% \mathrm{~N} \text { through Farm yard } \\
\text { manure (FYM) }(16.0 \mathrm{t} / \mathrm{ha})\end{array}$ & 0.88 & 0.31 & 0.76 \\
\hline $\mathbf{T}_{9}$ & $\begin{array}{l}100 \% \mathrm{~N} \text { through Poultry } \\
\text { manure }(\mathrm{PM})(5.0 \mathrm{t} / \mathrm{ha})\end{array}$ & 0.85 & 0.29 & 0.73 \\
\hline $\mathbf{T}_{10}$ & $\begin{array}{l}100 \% \quad \mathrm{~N} \quad \text { through } \\
\text { Vermicompost (VC) }(7.0 \mathrm{t} / \mathrm{ha})\end{array}$ & 1.06 & 0.38 & 0.92 \\
\hline \multirow[t]{4}{*}{$\mathbf{T}_{11}$} & $\begin{array}{l}\text { Control (without inorganic or } \\
\text { organic sources of nutrition) }\end{array}$ & 0.51 & 0.17 & 0.43 \\
\hline & C.D. & 0.37 & 0.14 & 0.33 \\
\hline & SE(m) & 0.12 & 0.05 & 0.11 \\
\hline & C.V. & 16.16 & 16.59 & 16.30 \\
\hline
\end{tabular}

\section{Nitrogen ( $N$ ) content (percent)}

The use of inorganic fertilizers and organic manures or micronutrients was found significant ranged between 1.51 to 1.63 percent. The higher concentration was found in treatment $50 \%$ RDF inorganic $+50 \% \mathrm{~N}$ through Vermicompost $(\mathrm{VC})\left(\mathrm{T}_{4}\right)$ and it was at par with $\mathrm{T}_{2}, \mathrm{~T}_{3}$ and $\mathrm{T}_{1}$. The lower concentration $(1.51 \%)$ was found in treatment $\mathrm{T}_{11}$.
Phosphorus $\left(\mathbf{P}_{2} \mathrm{O}_{5}\right)$ content $(\%)$

The result was found significant during the study, pertaining to $\mathrm{P}_{2} \mathrm{O}_{5}$ content in pod. It was significantly noticed that $\mathrm{P}_{2} \mathrm{O}_{5}$ content ranged from $0.50\left(\mathrm{~T}_{11}\right)$ to $0.63\left(\mathrm{~T}_{4}\right)$ per cent. The higher $\mathrm{P}_{2} \mathrm{O}_{5}$ content $(0.63 \%)$ was observed in treatment $\mathrm{T}_{4}$ which was at par with the treatments $T_{2}(0.62), T_{3}(0.61)$ and $T_{1}$ $(0.60)$ where as the lower phosphorus content $(0.50 \%)$ was observed in treatment $T_{11}$. 


\section{Potassium $\left(\mathrm{K}_{2} \mathrm{O}\right)$ content $(\%)$}

Perusal the data given treatment effect was found significant at five percent level of significance. The maximum $\mathrm{K}_{2} \mathrm{O}$ content was found in treatment $\mathrm{T}_{4}(1.46 \%)$, during the crop study period. The minimum $\mathrm{K}_{2} \mathrm{O}$ content was found in treatment $\mathrm{T}_{11}(1.30 \%)$.

\section{Nutrient uptake by Okra pod}

\section{Nitrogen uptake $\left(\mathrm{kg} \mathrm{ha}^{-1}\right)$}

During the investigation, the highest mean value of $2.22 \mathrm{~kg} / \mathrm{ha} \mathrm{N}$ uptake by okra pod was registered, with the treatment $\mathrm{T}_{4}(50 \% \mathrm{RDF}$ inorganic $+50 \% \mathrm{~N}$ through Vermicompost (VC)). The lowest value of $0.51 \mathrm{~kg} / \mathrm{ha}$ was found in pod with treatment $\mathrm{T}_{11}$.

\section{Phosphorus uptake (kg ha $\left.{ }^{-1}\right)$}

Phosphorus $\left(\mathrm{P}_{2} \mathrm{O}_{5}\right)$ uptake in course of investigation, the higher $\mathrm{P}_{2} \mathrm{O}_{5}$ uptake in pod was $0.85 \mathrm{~kg} / \mathrm{ha}\left(\mathrm{T}_{4}\right)$ and minimum uptake in pod was $0.17 \mathrm{~kg} / \mathrm{ha}$ in $\mathrm{T}_{11}$.

\section{Potassium $\left(\mathrm{K}_{2} \mathrm{O}\right)$ uptake $(\mathrm{kg} / \mathrm{ha})$}

During the investigation, application of $50 \%$ $\mathrm{RDF}$ inorganic $+50 \% \mathrm{~N}$ through Vermicompost (VC) $\left(\mathrm{T}_{4}\right)$ showed higher uptake of $\mathrm{K}_{2} \mathrm{O}$ by pod. The application of control $\left(\mathrm{T}_{11}\right)$ showed relatively lower uptake of $\mathrm{K}_{2} \mathrm{O}$ by pods.

\section{References}

Ahmed N and Tanki M I, 1997. Effect of nitrogen and phosphorus on growth and Seed yield of okra. Indian Journal of Horticulture 54(2): 156-159.

Ansari AA and Kumarsukhraj, 2010. Effect of vermiwash and vermicompost on soil parameters and productivity of Okra (Abelmoschus esculentus) in Guyana. African Journal of Agricultural Research 5(14): 1794-1798.

Barani P and Anburani A, 2004 Influence of vermicomposting on major nutrients in bhendi (Abelmoschus esculentus(L.)Moench) var. Arka Anamika. South Indian Horticulture 52(1-6): 351-354.

Bharthy, R. Bhu, Sankaran, M. and Subramani, T., 2017. Effect of integrated nutrient management on nutrient uptake and yield of okra [Abelmoschus esculentus (L.) Moench] under islands conditions. Adv. Res. J. Crop Improv., 8 (1): 24-30.

Miller M H, Mitchell W A, Stypa M and Barry D A, 1987. Effects of nutrient availability and subsoil bulk density on corn yield and nutrient absorption. Canadian Journal, Soil Science 67:28192.

Naik L B and Srinivas K, 1992. Influence of $\mathrm{N}$ and $\mathrm{P}$ fertilization on seed crop of okra. Indian J. Agron. 37: 769-71.

Olsen S R, 1954. Estimation of available phosphorous in soil by extraction with sodium bicarbonate.US department of agriculture, Washington D.C circular no. 939.

Parvatham A, Vijayan K P and Nazar A, 1989. Effect of Azospirillum on growth and nutrient uptake of Pusa Sawani bhendi (Abelmoschus esculentus L. Moench). South Indian Horticulture 37(4): 227-229.

Yadav G. L., Singh S. P., Jitarwal O. P., Yadav V. K. and Choudhary R., 2017. Effect of Nitrogen and Bio-organics on Growth and Yield of Okra [Abelmoschus esculentus (L.) Moench]. Chem Sci Rev Lett, 6(23), 1515-1519. 


\section{How to cite this article:}

Veer Bhan Singh, Anil Kumar Tiwari. 2019. Effect of Integrated Nutrient Management (INM) on Physico-Chemical Properties of Soil, Available Content and Nutrient Uptake by Okra (Abelmoschus esculentus). Int.J.Curr.Microbiol.App.Sci. 8(03): 130-137. doi: https://doi.org/10.20546/ijcmas.2019.803.018 